Rev. bras. zootec., v.28, n.3, p.495-503, 1999

\title{
Análise Comparativa de Procedimentos Metodológicos para a Avaliação Genética de Vacas para a Produção de Leite
}

\author{
Mario Luiz Martinez ${ }^{1,2}$, Rui da Silva Verneque ${ }^{1,2}$, Roberto Luiz Teodoro ${ }^{1,2}$, Amaury Alipio \\ Pimentel $^{2}$, William José Ferreira ${ }^{2}$
}

RESUMO - Registros de produção de leite de 19.151 lactações de 8.984 vacas, filhas de 795 touros Gir, foram usados para avaliar geneticamente as vacas. Equação de modelo misto foi usada para predizer o valor genético dos touros que foram incorporados no índice de seleção das vacas. Além do índice de seleção, os modelos animais foram também usados para predizer o valor genético das vacas, usando somente a primeira lactação ou até cinco lactações por vaca. As confiabilidades das predições obtidas dos valores genéticos das vacas usando-se os modelos animais foram maiores, quando foram usadas na avaliação até cinco lactações $(0,76)$ ou somente a primeira lactação $(0,73)$, em relação às confiabilidades das predições obtidas pelos índices de seleção $(0,55$ a 0,59$)$. Semelhantemente, as correlações entre os valores genéticos preditos pelos índices de seleção e os preditos pelo modelo animal com até cinco lactações variaram de 0,74 a 0,84. A maior correlação $(0,84)$ foi observada entre as predições obtidas pelos modelos animal e a menor, entre o modelo animal com até cinco lactações e o índice de seleção, no qual os valores genéticos dos pais foram obtidos pelo método das companheiras de rebanho. O ganho genético obtido quando se selecionaram $20 \%$ das melhores vacas, para produzir descendentes para a próxima geração, foi de, no mínimo, $80 \%$ do ganho obtido pelo modelo animal $(270 \mathrm{~kg})$, independente do procedimento usado.

Palavras-chave: avaliação de vacas, correlação, metodologia, confiabilidade

\section{Comparative Analyses among Methodological Procedures for Genetic Evaluation of Milk Production Cows}

\begin{abstract}
Milk production data of 19,151 lactations from 8,984 cows, daughters of 795 Gyr sires were used to genetically evaluate cows. Mixed model equation was used to predict sire-breeding values that were incorporated in the selection index of the cows. Besides the selection index, animal models were also used to predict the genetic value of the cows, using only the first lactation or up to five lactations per cow. The accuracies of the predictions obtained by cows breeding values, using the animal models, were higher when were used in the evaluation up to five lactations (.76) or only the first lactation (.73) on the accuracies predictions obtained by the selection index (.55 to .59). Similarly, the correlation among the breeding values predicted by selection index and predicted by animal model up to five lactations ranged from .74 to .84 . The highest correlation (.84) was observed among the prediction obtained by animal models and the lowest (.74) within the animal model with up to five lactations and the selection index, which the sire-dam breeding values were obtained by the herdmate method. The genetic gain obtained when $20 \%$ of best cows were selected to produce descendants for the new generation was at least $80 \%$ of the gain obtained by animal model $(270 \mathrm{~kg})$, regardless the used procedure.
\end{abstract}

Key Words: cow evaluation, correlation, methodology, accuracy

\section{Introdução}

Diversos são os procedimentos disponíveis para predizer o valor genético para produção de leite das fêmeas bovinas (HILL e SWANSON, 1983; POWELL et al., 1983; e NORMAN et al., 1991). A diferença básica entre os procedimentos está relacionada principalmente à quantidade de informação utilizada e à flexibilidade de se realizarem avaliações mais freqüentes, sem ter que se manipular grande número de informações. A forma mais simplificada de se avaliar geneticamente uma vaca é multiplicar a sua produção, ajustada para efeitos não-genéticos, pela herdabilidade da característica (FALCONER, 1981). Uma série de procedimentos é derivada a partir deste caso mais elementar, considerando-se mais lactações de uma mesma vaca e informações de parentes (SPIKE e FREEMAN, 1978). Em qualquer caso, o objetivo principal de qualquer avaliação é obter predição precisa e não-viciada do valor genético do indivíduo, de maneira a se maximizar o ganho genético (HENDERSON, 1975).

SPIKE e FREEMAN (1978) usaram o índice de seleção para obter quatro predições do valor genético 
das vacas, usando informações das próprias vacas e de suas meio-irmãs paternas, suas mães, meio-irmãs maternas e filhas. Esses autores concluíram que a inclusão de informações dos parentes e o ajuste para a média genética do rebanho melhoraram a associação das predições dos valores genéticos com a produção do rebanho e que as correlações entre os valores genéticos obtidos foram todas maiores que 0,88 .

Segundo DOMMERHOLT (1980), embora a produção varie mais em rebanhos de alta produção, o coeficiente de variação é semelhante para todos os níveis de produção e a relação positiva entre médias e variâncias aumenta a probabilidade de uma vaca ser considerada elite em um rebanho de alta produção. Assim, os desvios das produções em relação à média, em rebanhos de alta produção, devem ser ponderados por um fator maior, relativo aos desvios provenientes dos rebanhos de baixa produção. Os procedimentos que utilizam o índice de seleção para estimar o valor genético das vacas requerem o pré-ajustamento dos dados para os efeitos não-genéticos. Embora de fácil aplicação e computacionalmente simples, o índice pode apresentar predições viciadas devido a não-eliminação total dos efeitos não-genéticos.

As equações dos modelos mistos (EMM; HENDERSON, 1973) que ajustam simultaneamente para os efeitos não-genéticos, ao mesmo tempo em que prediz os valores genéticos das vacas, pelo uso do modelo animal (HENDERSON, 1988; VAN VLECK, 1992), apresentam alto requerimento de memória de computador e de tempo de processamento, principalmente quando se avalia grande número de animais e com muitas classes de efeitos fixos.

Uma alternativa que permite combinar as vantagens dos modelos mistos e dos índices de seleção é o procedimento no qual os valores genéticos dos touros são preditos pelas equações dos modelos mistos (HENDERSON, 1988) e incorporados no índice de seleção para predizer os valores genéticos das vacas. Este procedimento pode ser usado quando o volume de dados e o custo de processamento dificultam o uso da EMM, que simultaneamente prediz os valores genéticos de touros e vacas e permite empregar qualquer número de lactações de uma vaca no cálculo de seu índice genético, independente do número de lactações utilizadas na avaliação dos touros. HILL e SWANSON (1983) desenvolveram um índice que combinava a primeira e as demais lactações da vaca, os valores genéticos dos pais e o valor genético do rebanho. Nesse índice, assumiram que a correlação genética entre a primeira lactação e as demais era diferente da unidade, mas que as correlações genéticas entre as demais lactações $\left(2^{\mathrm{a}}, 3^{\mathrm{a}}, 4^{\mathrm{a}}\right.$ etc $)$ eram iguais à unidade. NORMAN et al. (1991) realizaram comparações entre o modelo animal e um índice obtido pelo método das comparações contemporâneas modificadas (DICKINSON et al., 1976) e encontraram correlações entre as avaliações genéticas de vacas variando de 0,92 a 0,96, dependendo da raça estudada. Verificaram também que as confiabilidades das predições dos valores genéticos foram apenas ligeiramente maiores $(0,02 \mathrm{a}$ $0,05)$ para o modelo animal.

O objetivo deste trabalho foi comparar procedimentos para avaliar geneticamente vacas, considerando-se as equações do modelo misto para predizer o valor genético dos touros e a incorporação desses valores em um índice de seleção para vacas que incluiu informações de até cinco lactações.

\section{Material e Métodos}

Os dados utilizados neste trabalho são provenientes de rebanhos Gir e mestiços Gir:Holandês que participam do programa de teste de progênie de touros da raça Gir coordenado pela Embrapa Gado de Leite em convênio com a Associação Brasileira de Criadores de Gir Leiteiro (ABCGIL). Um total de 19.151 lactações, de 8984 vacas que pariram entre 1965 e 1996, filhas de 795 touros, foi usado para se estimar o valor genético para produção de leite em até 305 dias de lactação.

Os procedimentos utilizados para a avaliação genética das vacas foram:

1) Equações dos modelos mistos e uso do modelo animal (MA) considerando-se até as cinco primeiras lactações da vaca e toda a matriz de parentesco.

O modelo estatístico adotado foi:

$$
y=\mathrm{X} \beta+\mathrm{Z} u+\mathrm{Z} p+\varepsilon
$$

em que y é um vetor que contém as produções de leite de cada vaca, em cada lactação; $X$, a matriz de incidência dos efeitos fixos (rebanho-ano, época do parto, composição genética e idade da vaca ao parto); $\beta$, um vetor de parâmetros desconhecidos representando os efeitos fixos; $Z$, a matriz de incidência dos efeitos aleatórios; $\mu$, vetor de efeitos aleatórios, que inclui os valores genéticos preditos dos animais; $p$, o vetor de efeito permanente de meio, decorrente de registros repetidos de uma mesma vaca; e $\varepsilon$, o vetor dos erros aleatórios.

As épocas de parto consideradas foram: 1- partos de outubro a março e 2 - partos de abril a setembro com, respectivamente, 2571 e 2594 observações. Para a 
composição genética consideraram-se os seguintes grupos: animais Gir, 1/8 Holandês-Gir, 1/4 Holandês-Gir, 3/8 Holandês-Gir e 1/2 Holandês-Gir, com 4050, 29, 227,555 e 304 observações, respectivamente. O número de subclasses rebanho-ano foi de 408.

Os valores genéticos para cada animal foram preditos usando-se o sistema MTDFREML (BOLDMAN et al., 1995). Por este sistema, os valores genéticos são preditos por um processo que envolve dois passos:

a) Estimação dos componentes de variância por meio da maximização da função de verossimilhança restrita do vetor y (método da máxima verossimilhança restrita - REML);

b) Conhecidas as estimativas dos componentes de variância, estas são substituídas nas equações do modelo misto, que são resolvidas por intermédio da decomposição de Cholesky (BOLDMAN et al., 1995; VERNEQUE, 1994), dispensando o uso de inversas de vacas. As soluções para o vetor u são os valores genéticos preditos dos animais.

A confiabilidade (REL) para cada valor genético foi estimada, segundo VAN VLECK (1993), pela expressão:

em que

$$
R E L=\sqrt{1-\frac{P E V}{\hat{\sigma}_{a}^{2}(1+F A)}}
$$

$\hat{\sigma}_{a}^{2}$ é a estimativa da variância dos efeitos genéticos aditivos; FA, o coeficiente de consangüinidade do animal, cujo valor genético está sendo predito; PEV, a variância do erro de predição do valor genético do animal, calculada como: $\mathrm{PEV}=\mathrm{C}^{-1} \mathrm{k}$, com $\mathrm{C}^{-1}$ a inversa da matriz de coeficientes das equações do modelo misto; e k, um vetor de mesma ordem da matriz C, com 1 na linha correspondente ao animal e 0 nas demais linhas.

2) Uso do modelo animal considerando-se apenas a produção no primeiro parto de cada vaca (MA1). Neste caso o MA foi utilizado, eliminado-se a componente $Z p$ da equação 1 .

Em ambas as análises com o modelo, solicitou-se que as vacas tivessem produção em seu primeiro parto. Assim, o número de vacas avaliadas pelo MA e MA1 foi de 5165.

3) Uso do índice de seleção (I) calculado conforme a seguinte expressão:

$$
\mathrm{I}=b_{1} D V+b_{2} V G_{t}+b_{3} V G_{m}+b_{4} R A
$$

em que

$$
\begin{gathered}
b_{1} D V=b_{11} D V_{1}+b_{12} D V_{2}+b_{13} D V_{3}+b_{14} D V_{4}+b_{15} D V_{5} \\
D V_{i}=\frac{n_{j}}{n_{j}-1}\left(\frac{P_{c i}-A H M_{j}}{A H M_{j}}\right) * M
\end{gathered}
$$

$$
A H M_{j}=M+\frac{n_{j}}{n_{j}+1}\left(H M_{j}\right)
$$

em que $\mathrm{P}_{c i}$ é a produção de leite na lactação ajustada para os efeitos de idade da vaca ao parto e composição genética; $n_{j}$, o número de vacas utilizadas para calcular a média de determinada classe de rebanho-ano-estação ${ }_{j}\left(H M_{j}\right) ; \mathrm{M}$, a média geral da população analisada; $A H M_{j}$, a média de todas as lactações ajustadas iniciadas na subclasse $H M_{j} ; D V_{i}$, a produção da vaca na lactação i, ajustada para a média da subclasse $A H M_{j} ; V G_{t}$, o valor genético do pai da vaca estimado por intermédio do método das companheiras contemporâneas (DOMMERHOLT, 1982); $V G_{m}$, o valor genético da mãe da vaca, estimado pela equação 2; RA, a média genética das companheiras de rebanho, estimada como sendo a metade dos valores genéticos dos pais das companheiras de rebanho; e os b's são coeficientes de regressão parciais que variam segundo a informação disponível, a confiabilidade das estimativas do valor genético do pai e da mãe e as correlações genéticas e fenotípicas entre as lactações e as herdabilidades consideradas para a produção de leite em cada característica. O coeficiente de regressão para ponderar a média genética das companheiras de rebanho é calculado como $b_{4}$ $=1-b_{2}-b_{3}$ (MOEN, 1978), em que $b_{2}$ e $b_{3}$ são os coeficientes de regressão para os valores genéticos dos pais e das mães das vacas. As herdabilidades e as correlações genéticas e fenotípicas utilizadas para o cálculo dos coeficientes de regressão parciais foram obtidas de DOMMERHOLT (1982) e estão na Tabela 1. Os coeficientes parciais de regressão foram calculados por meio da resolução do sistema de equações:

$\mathrm{Cb}=\mathrm{G}$ que pode ser escrito como:

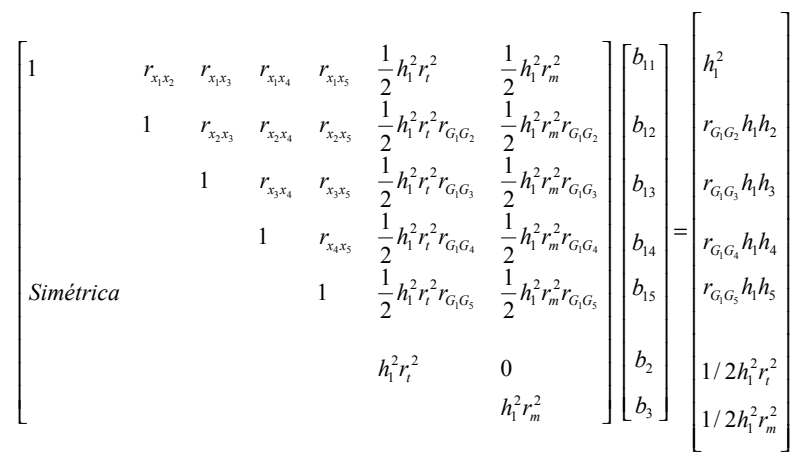

em que $r_{x_{i} x_{j}}$ e $r_{G_{i} G_{j}}$ são, respectivamente, as correlações fenotípicas e genéticas para produção de leite entre as lactações i e j; $h_{i}^{2}$ é a herdabilidade da produção de leite para a lactação i; e $r_{t}$ e $r_{m}$ são as confiablidades das estimativas dos valores genéticos 
Tabela 1 - Herdabilidades (na diagonal) e correlações genéticas (acima da diagonal) e fenotípicas (abaixo da diagonal) para a produção de leite nas primeiras cinco lactações

Table 1 - Heritabilities (on diagonal), genetic (upperdiagonal) and phenotypic (lower diagonal) correlations formilkyield in the first five lactations

\begin{tabular}{lccccc}
\hline Lactação & \multicolumn{5}{c}{$\begin{array}{c}\text { Lactação } \\
\text { Lactation }\end{array}$} \\
\cline { 2 - 6 } & 1 & 2 & 3 & 4 & 5 \\
\hline 1 & 0,25 & 0,85 & 0,85 & 0,85 & 0,85 \\
2 & 0,55 & 0,20 & 0,85 & 0,85 & 0,85 \\
3 & 0,50 & 0,60 & 0,20 & 0,85 & 0,85 \\
4 & 0,45 & 0,55 & 0,60 & 0,20 & 0,85 \\
5 & 0,40 & 0,50 & 0,55 & 0,60 & 0,20 \\
\hline
\end{tabular}

dos touros e das mães. A solução para este sistema de equações foi obtida considerando-se os valores da Tabela 1 e assumindo-se confiabilidade média do valor genético das mães de $0,6\left(r_{m}\right)$ e três valores de confiabilidade para o valor genético dos touros $\left(r_{t}=\right.$ $0,5,0,8$ e 0,91 ) baixo, médio e alto. Os valores dos $b$ 's, utilizados para se calcular o valor genético pelo índice para as diversas situações, e a confiabilidade $\left(r_{i}\right)$ das predições dos valores genéticos estão na Tabela 2 .

4) Uso do índice de seleção conforme a equação 2 , porém com valor genético do touro predito pelo modelo animal usando-se as produções nos cinco

Tabela 2 - Coeficiente de regressão (b) usado no índice de seleção em função da informação disponível

Table 2 - Regression coefficient (b) used in the selection index on the available information

\begin{tabular}{|c|c|c|c|c|c|c|c|c|}
\hline & \multicolumn{5}{|c|}{$\begin{array}{r}\text { Vaca } \\
\text { Cow }\end{array}$} & \multirow{2}{*}{$\begin{array}{c}\text { Touro } \\
\text { Sire } \\
\mathrm{b}_{2}\end{array}$} & \multirow{2}{*}{$\begin{array}{c}\text { Mãe } \\
\text { Dam } \\
\mathrm{b}_{3}\end{array}$} & \multirow[t]{2}{*}{$\mathrm{r}_{\mathrm{I}}$} \\
\hline & $\mathrm{b}_{11}$ & $\mathrm{~b}_{12}$ & $\mathrm{~b}_{13}$ & $\mathrm{~b}_{14}$ & $\mathrm{~b}_{15}$ & & & \\
\hline & 0,2500 & & & & & & & 0,500 \\
\hline & 0,2086 & 0,0754 & & & & & & 0,516 \\
\hline Apenas a vaca & 0,1920 & 0,0438 & 0,0678 & & & & & 0,526 \\
\hline \multirow[t]{4}{*}{ Only cow } & 0,1842 & 0,0267 & 0,0413 & 0,0676 & & & & 0,536 \\
\hline & 0,1810 & 0,0191 & 0,0258 & 0,0418 & 0,0698 & & & 0,546 \\
\hline & 0,2327 & & & & & & 0,3836 & 0,549 \\
\hline & 0,1957 & 0,0681 & & & & & 0,3732 & 0,561 \\
\hline Vaca+mãe & 0,1809 & 0,0394 & 0,0620 & & & & 0,3665 & 0,569 \\
\hline \multirow[t]{4}{*}{ Cow +dam } & 0,1739 & 0,0237 & 0,0376 & 0,0623 & & & 0,3605 & 0,577 \\
\hline & 0,1710 & 0,0149 & 0,0234 & 0,0385 & 0,0647 & & 0,3544 & 0,585 \\
\hline & 0,2381 & & & & & 0,3810 & & 0,535 \\
\hline & 0,1997 & 0,0704 & & & & 0,3702 & & 0,547 \\
\hline Vaca $^{1}+$ touro 1 & 0,1843 & 0,0408 & 0,0638 & & & 0,3634 & & 0,556 \\
\hline \multirow[t]{4}{*}{ Cow + sire 1} & 0,1771 & 0,0247 & 0,0388 & 0,0640 & & 0,3573 & & 0,564 \\
\hline & 0,1741 & 0,0156 & 0,0241 & 0,0396 & 0,0663 & 0,3512 & & 0,573 \\
\hline & 0,2188 & & & & & 0,3906 & & 0,586 \\
\hline & 0,1853 & 0,0623 & & & & 0,3809 & & 0,595 \\
\hline Vaca $^{1}+$ touro 2 & 0,1717 & 0,0358 & 0,0573 & & & 0,3746 & & 0,602 \\
\hline \multirow[t]{4}{*}{ Cow + sire 2} & 0,1653 & 0,0213 & 0,0347 & 0,0580 & & 0,3689 & & 0,608 \\
\hline & 0,1628 & 0,0130 & 0,0214 & 0,0358 & 0,0604 & 0,3631 & & 0,615 \\
\hline & 0,2091 & & & & & 0,3955 & & 0,611 \\
\hline & 0,1780 & 0,0582 & & & & 0,3836 & & 0,618 \\
\hline Vaca+touro3 & 0,1653 & 0,0333 & 0,0540 & & & 0,3802 & & 0,624 \\
\hline \multirow[t]{4}{*}{ Cow + sire 3} & 0,1594 & 0,0195 & 0,0326 & 0,0550 & & 0,3748 & & 0,629 \\
\hline & 0,1571 & 0,0117 & 0,0200 & 0,0339 & 0,0575 & 0,3691 & & 0,635 \\
\hline & 0,2203 & & & & & 0,3899 & 0,3899 & 0,582 \\
\hline & 0,1864 & 0,0629 & & & & 0,3801 & 0,3801 & 0,592 \\
\hline Vaca+mãe+touro1 & 0,1727 & 0,0362 & 0,0578 & & & 0,3737 & 0,3737 & 0,598 \\
\hline \multirow[t]{4}{*}{ Cow + dam+sirel } & 0,1663 & 0,0216 & 0,0350 & 0,0585 & & 0,3680 & 0,3680 & 0,605 \\
\hline & 0,1638 & 0,0133 & 0,0216 & 0,0361 & 0,0610 & 0,3621 & 0,3621 & 0,612 \\
\hline & 0,2000 & & & & & 0,4000 & 0,4000 & 0,632 \\
\hline & 0,1712 & 0,0543 & & & & 0,3913 & 0,3913 & 0,639 \\
\hline Vaca+mãe+touro2 & 0,1594 & 0,0309 & 0,0509 & & & 0,3856 & 0,3856 & 0,644 \\
\hline \multirow[t]{4}{*}{ Cow + dam + sire 2} & 0,1538 & 0,0179 & 0,0306 & 0,0521 & & 0,3803 & 0,3803 & 0,648 \\
\hline & 0,1517 & 0,0105 & 0,0187 & 0,0321 & 0,0547 & 0,3749 & 0,3749 & 0,654 \\
\hline & 0,1898 & & & & & 0,4051 & 0,4051 & 0,656 \\
\hline & 0,1635 & 0,0500 & & & & 0,3970 & 0,3970 & 0,661 \\
\hline Vaca+mãe+touro3 & 0,1526 & 0,0283 & 0,0474 & & & 0,3915 & 0,3915 & 0,665 \\
\hline \multirow[t]{2}{*}{ Cow + dam+sire 3} & 0,1475 & 0,1616 & 0,0284 & 0,0489 & & 0,3865 & 0,3865 & 0,669 \\
\hline & 0,1456 & 0,0092 & 0,0172 & 0,0301 & 0,0516 & 0,3813 & 0,3813 & 0,674 \\
\hline
\end{tabular}

${ }^{1}$ Touro 1 = baixa confiabilidade na sua avaliação genética $\left(r_{t}=0,50\right)$; Touro $2=$ média confiabilidade $\left(r_{t}=0,80\right)$; Touro $3=$ alta confiabilidade $\left(r_{t}=0,91\right)$.

${ }^{1}$ Sire $1=$ low accuracy in the genetic evaluation $\left(r_{t}=.50\right)$; Sire $2=$ average accuracy $\left(r_{t}=.80\right)$; Sire $3=$ high accuracy $\left(r_{t}=.91\right)$. 
primeiros partos de cada vaca $\left(\mathrm{I}_{\mathrm{AMT}}\right)$.

5) Uso do índice de seleção conforme a equação 2 , porém com valor genético do touro predito pelo modelo animal usando-se a produção no primeiro parto de cada vaca $\left(\mathrm{I}_{\mathrm{A} 11}\right)$.

6) Uso do índice de seleção conforme a equação 2 , porém com valor genético do touro predito pelas equações dos modelos mistos (equação 1), usando-se o modelo de touros e as produções nos cinco primeiros partos de cada vaca $\left(\mathrm{I}_{\mathrm{ATT}}\right)$.

7) Uso do índice de seleção conforme a equação 2, porém com os valores genéticos do touro e da mãe preditos pelo modelo animal (equação 1) utilizando-se as produções nos cinco primeiras partos de cada vaca $\left(\mathrm{I}_{\mathrm{PMT}}\right)$.

Em todas as situações em que o índice foi utilizado, solicitou-se que as vacas tivessem produção em seu primeiro parto, ou seja, a primeira lactação não foi necessariamente a do primeiro parto. Dessa forma, foram avaliadas as 8984 vacas existentes no arquivo de dados.

Os procedimentos foram comparados por intermédio das correlações de ordem (método de Spearman) e de valores (método de Pearson) (CONOVER, 1980), da variação dos valores genéticos, da confiabilidade das estimativas, do desvio médio absoluto entre os valores genéticos estimados por MA e os valores genéticos obtidos pelos demais procedimentos, da porcentagem de coincidência na seleção das mesmas vacas pelos diferentes procedimentos com relação às vacas selecionadas pelo modelo animal e da resposta espera- da à seleção para a produção de leite calculada com base nas estimativas obtidas por intermédio dos procedimentos utilizados para a medição dos valores genéticos das vacas.

\section{Resultados e Discussão}

As médias, os desvios-padrão, a confiabilidade e a variação dos valores genéticos preditos pelos diferentes procedimentos são apresentados na Tabela 3 . Pode-se observar que as maiores médias dos valores genéticos, as maiores confiabilidades e as menores amplitudes de variação dos valores genéticos foram obtidas por intermédio dos procedimentos MA e MA1, que utilizaram o modelo animal. A confiabilidade das predições obtidas pelo MA foi no mínimo 0,17 unidades maior que qualquer um dos outros procedimentos. Essa confiabilidade, assim como a média dos valores genéticos, foi ligeiramente menor, quando apenas a primeira lactação das vacas foi considerada (MA1). Apesar de ser explicável menor confiabilidade pelo uso de menor número de informações, não é claro porque a média diminuiu, visto que as produções foram simultaneamente ajustadas para os efeitos nãogenéticos com o uso do modelo animal. Possível explicação pode ser o fato de que no MA usaram-se outras lactações além da primeira que, possivelmente, estariam influenciadas por algum tratamento preferencial que não foi corrigido pelo modelo.

Quando se compararam os valores obtidos por

Tabela 3 - Média, desvio-padrão, confiabilidade e amplitude dos valores genéticos preditos por diferentes procedimentos Table 3 - Mean, standard deviation, accuracy and range of predicted genetic values by different procedures

\begin{tabular}{lccccc} 
Procedimento $^{1}$ & $\begin{array}{c}\text { N }^{\text {de vacas }} \\
\text { Nrocedure }\end{array}$ & $\begin{array}{c}\text { Média }(\mathrm{kg}) \\
\text { Mean }\end{array}$ & $\begin{array}{c}\text { Desvio-padrão }(\mathrm{kg}) \\
\text { Standard deviation }\end{array}$ & $\begin{array}{c}\text { Confiabilidade } \\
\text { Accuracy }\end{array}$ & $\begin{array}{c}\text { Amplitude } \\
\text { Range }\end{array}$ \\
\hline MA & 5165 & 109 & 254 & 0,76 & -730 a 1354 \\
MA1 & 5165 & 76 & 214 & 0,73 & -811 a 1147 \\
I & 8984 & -11 & 280 & 0,55 & -1200 a 2799 \\
I $_{\text {AMT }}$ & 8984 & 42 & 300 & 0,59 & -1267 a 2071 \\
I $_{\text {ATT }}$ & 8984 & 34 & 296 & 0,59 & -1261 a 2034 \\
I $_{\text {A11 }}$ & 8984 & 24 & 282 & 0,58 & -1192 a 1764 \\
I $_{\text {PMT }}$ & 8984 & 43 & 299 & 0,59 & -1267 a 1503 \\
\hline
\end{tabular}

${ }^{1} \mathrm{MA}$ Avaliação genética pelo modelo animal considerando-se até as cinco primeiras lactações.

MA1 Idem ao MA, porém utilizando-se apenas a primeira lactação.

I Avaliação pelo índice com até as cinco primeiras lactações, valor genético $\left(V G_{t}\right)$ do pai estimado pelo método das companheiras contemporâneas e o valor genético da mãe $\left(V G_{m}\right)$ calculado pelo índice utilizando-se até as cinco primeiras lactações da mãe.

$\mathrm{I}_{\mathrm{AMT}}$ Avaliação pelo índice, porém com o $\mathrm{VG}_{\mathrm{t}}$ calculado pelo modelo animal utilizando-se as primeiras cinco lactações de cada vaca.

$\mathrm{I}_{\mathrm{ATT}}$ Idem ao I $\mathrm{AMT}$, porém com o $\mathrm{VG}_{\mathrm{t}}$ calculado pelo modelo de touro.

$\mathrm{I}_{\mathrm{A} 11}$ Idem ao I $\mathrm{AMT}$, porém com o $\mathrm{VG}_{\mathrm{t}}$ calculado utilizando-se apenas a primeira lactação de cada vaca.

IPMT Avaliação pelo índice, porém com os $V G_{t}$ e $V G_{m}$ calculados pelo modelo animal utilizando-se as primeiras cinco lactações de cada vaca.

MA Genetic evaluation using animal model with up to five lactations by cow.

MA1 As MA, but with only the first lactation;

I Evaluation by the selection index with up to five lactations of the cow, breeding value of sire $\left(V G_{t}\right)$ estimated by herdmate comparison and the breeding value of the dam $\left(V G_{m}\right)$ estimated by the selection index with up to five lactations.

$I_{A M T}$ Evaluation by the index, but $V G_{t}$ estimated by animal model with up to five lactations per cow.

$I_{A T T}$ As $I_{A M T}$, but $V G_{t}$ estimated by sire model.

$I_{A 11}$ As $I_{A M T}$, but $V G_{t}$ estimated by animal model with only first lactation.

$I_{P M T}$ Evaluation by the index, but $V G_{t}$ and $V G_{m}$ estimated by animal model with up to five lactations per cow. 
meio dos índices de seleção (I, $\mathrm{I}_{\mathrm{AMT}}, \mathrm{I}_{\mathrm{ATT}}, \mathrm{I}_{\mathrm{A} 11} \mathrm{e}$ $\mathrm{I}_{\mathrm{PMT}}$ ), observou-se pequeno ganho em confiabilidade quando os valores genéticos dos touros foram preditos pelo modelo animal. Verificou-se ainda que no procedimento $\mathrm{I}_{\mathrm{A} 11}$, no qual o $\mathrm{VG}$ dos touros foi predito considerando-se apenas a produção no primeiro parto das vacas, a confiabilidade $(0,58)$ foi praticamente igual à dos demais procedimentos $\left(\mathrm{I}_{\mathrm{AMT}}, \mathrm{I}_{\mathrm{ATT}} \mathrm{I}_{\mathrm{PMT}}\right)$, em que o VG dos touros foi predito empregando-se mais de uma lactação de cada vaca. Faz-se importante considerar nessas comparações que as estimativas obtidas pelos índices de seleção são resultados não apenas das produções ajustadas de cada lactação, como também dos valores dos coeficientes de regressão parciais (Tabela 2), que foram calculados com base nos parâmetros apresentados na Tabela 1. Em contrapartida, nos resultados obtidos pelo modelo animal (MA e MA1), as estimativas de herdabilidade e repetibilidade foram calculadas simultaneamente à obtenção dos valores genéticos; conseqüentemente, estas estimativas refletem melhor a estrutura dos dados.
Quando se analisam os resultados (Tabela 4) estratificados por classe de confiabilidade, obtida por meio do índice I, observa-se que ocorreu aumento da média dos valores genéticos, quando a confiabilidade aumentou. As médias genéticas foram todas negativas quando a confiabilidade pelo I variou entre 0,40 e 0,50 , mesmo para as predições obtidas pelo modelo animal (MA e MA1), que proporcionaram confiabilidade maior $(0,68$ e 0,67$)$. Quando a confiabilidade pelo I variou de 0,60 a 0,70 , todas as médias foram positivas, com exceção da obtida pelo I, e relativamente altas quando comparadas às estimadas obtidas nas duas outras classes de confiabilidade.

O modelo animal permitiu a obtenção de predições de VG com confiabilidades que variaram de 0,68 a 0,79 , resultados superiores aos reportados por NORMAN et al. (1991), que variaram de 0,63 a 0,69, dependendo da raça estudada. Todavia, quando se compararam os resultados obtidos pelos índices, que variaram de 0,50 a 0,64 neste estudo, com os publicados por esses autores $(0,60$ a 0,67$)$ no método das

Tabela 4 - Média, desvio-padrão, confiabilidade e amplitude dos valores genéticos preditos por diferentes procedimentos para diferentes valores de confiabilidade do índice de seleção

Table 4 - Mean, standard deviation, accuracy and range of genetic values predicted by different procedures fordifferent values of accuracy of the selection index

\begin{tabular}{lccccc}
\hline Procedimento & $\mathrm{N}^{0}$ de vacas & Média $(\mathrm{kg})$ & Desvio-padrão $(\mathrm{kg})$ & Confiabilidade & Amplitude \\
Procedure $^{l}$ & N. of cows & Mean & Standard deviation & Accuracy & Range \\
\hline
\end{tabular}

a) Confiabilidade pelo I entre 0,40 e 0,50

MA $\quad 456$

MA1 453

I 1564

$\mathrm{I}_{\mathrm{AMT}} \quad 1564$

I 1564

$\mathrm{I}_{\mathrm{A} 11} \quad 1564$

$\mathrm{I}_{\mathrm{PMT}} \quad 1564$

MA $\quad 4208$

MA1 4186

I 6774

$\mathrm{I}_{\mathrm{AMT}} \quad 6774$

I

$\mathrm{I}_{\mathrm{A} 11} \quad 6774$

$\mathrm{I}_{\mathrm{PMT}}$

6774

MA

MA1

I

$\mathrm{I}_{\mathrm{AMT}}$

$\mathrm{I}_{\mathrm{ATT}}$

$\mathrm{I}_{\mathrm{A} 11}$

$\mathrm{I}_{\text {PMT }}$

${ }^{1}$ Vide Tabela 3.

${ }^{1}$ As Table 3.

529

526

646

646

646

646

646
Accuracy by I between .40 and .50

$\begin{array}{llll}-30 & 210 & 0,68 & -622 \text { a } 745 \\ -49 & 195 & 0,67 & -811 \text { a } 629 \\ -74 & 246 & 0,50 & -912 \text { a } 987 \\ -65 & 261 & 0,53 & -881 \text { a } 996 \\ -68 & 256 & 0,52 & -873 \text { a } 997 \\ -72 & 253 & 0,52 & -930 \text { a } 979 \\ -64 & 260 & 0,53 & -881 \text { a } 996\end{array}$

b) Confiabilidade pelo I entre 0,50 e 0,60

Accuracy by I between .50 and .60

\begin{tabular}{cccc}
109 & 252 & 0,77 & -730 a 1354 \\
78 & 210 & 0,73 & -740 a 1147 \\
4 & 289 & 0,55 & -1200 a 2799 \\
56 & 305 & 0,60 & -1267 a 2071 \\
50 & 303 & 0,60 & -1261 a 2034 \\
37 & 286 & 0,59 & -1192 a 1764 \\
57 & 305 & 0,60 & -1267 a 1503 \\
c) Confiabilidade pelo I entre 0,60 e 0,70 & \\
\multicolumn{4}{c}{ Accuracy by I between .60 and .70} \\
167 & 244 & 0,79 & -522 a 908 \\
-18 & 216 & 0,78 & -653 a 854 \\
155 & 237 & 0,63 & -706 a 1202 \\
116 & 259 & 0,63 & -588 a 1199 \\
117 & 255 & 0,63 & -585 a 1198 \\
155 & 256 & 0,63 & -754 a 1151 \\
& 259 & 0,64 & -588 a 1199
\end{tabular}

-588 a 1199 
Rev. bras. zootec.

companheiras contemporâneas modificado, verificou-se que as confiabilidades obtidas no presente trabalho foram ligeiramente menores. Um ponto importante a observar é que, independente da classe de confiabilidade, todas as predições de VG obtidas pelos procedimentos que utilizaram o índice de seleção proporcionaram praticamente a mesma confiabilidade, sendo também as médias dos valores genéticos similares, à exceção da estimada pelo I.

Embora as predições dos valores genéticos e as suas confiabilidades sejam de grande importância para aferir ganhos genéticos, é também importante conhecer a consistência das predições obtidas pelos diferentes procedimentos e as classificações das vacas por essas predições. Os coeficientes de correlação entre os valores preditos e entre as classificações são apresentados na Tabela 5. As correlações $(0,84$ e $0,83)$ entre as predições dos valores genéticos e as classificações entre os procedimentos pelo modelo animal (MA e MA1) foram as mais altas dentre todas. Embora a única diferença entre estes procedimentos seja apenas o uso de maior número de lactações por vaca (até cinco), as correlações se afastam da unidade em 16e 17\%. As menores correlações ocorreram entre as predições pelo I e pelo MA $(0,74$ e 0,70$)$, refletindo o menor uso da informação, quando não se utilizam todas as relações de parentesco existente entre os animais. Todavia, quando o índice de seleção $\left(\mathrm{I}_{\mathrm{AMT}}\right)$ utilizou o VG dos touros preditos pelo modelo animal, as correlações foram da ordem de 0,81 a 0,79 , apenas 0,03 e 0,04 unidades menores que as correlações entre

Tabela 5 - Coeficiente de correlação de valores (acima da diagonal) e correlação de ordem (abaixo da diagonal) entre os valores genéticos preditos por diferentes procedimentos

Table 5 - Correlation coefficient of values (upperdiagonal) and rank correlation (lowerdiagonal) among genetic values predicted by different procedures

\begin{tabular}{lccccccc}
\hline \multicolumn{7}{c}{ Procedimento $^{1}$} & \multicolumn{7}{c}{ Procedimento } \\
Procedure $^{l}$ & \multicolumn{7}{c}{ Procedure } \\
\cline { 2 - 8 } & MA & MA1 & I & $\mathrm{I}_{\text {AMT }}$ & $\mathrm{I}_{\text {ATT }}$ & $\mathrm{I}_{\text {A11 }}$ & $\mathrm{I}_{\text {PMT }}$ \\
\hline MA & 1,0 & 0,84 & 0,74 & 0,81 & 0,79 & 0,77 & 0,81 \\
MA1 & 0,83 & 1,0 & 0,69 & 0,75 & 0,73 & 0,81 & 0,75 \\
$\mathrm{I}$ & 0,70 & 0,66 & 1,0 & 0,87 & 0,88 & 0,89 & 0,88 \\
$\mathrm{I}_{\text {AMT }}$ & 0,79 & 0,73 & 0,88 & 1,0 & 0,99 & 0,95 & 0,99 \\
$\mathrm{I}_{\text {ATT }}$ & 0,77 & 0,71 & 0,89 & 0,98 & 1,0 & 0,94 & 0,98 \\
$\mathrm{I}_{\text {A11 }}$ & 0,75 & 0,79 & 0,88 & 0,94 & 0,94 & 1,0 & 0,95 \\
$\mathrm{I}_{\text {PMT }}$ & 0,79 & 0,73 & 0,87 & 0,99 & 0,98 & 0,94 & 1,0 \\
\hline
\end{tabular}

${ }^{1}$ Vide Tabela 3.

${ }^{1}$ As Table 3. o MA e MA1. Quando se utilizou no índice o VG do touro predito pelo MA1 (procedimento $\mathrm{I}_{\mathrm{A} 11}$ ), a correlação dos valores preditos por $\mathrm{I}_{\mathrm{A} 11}$ e MA foi 0,77 , ou seja, apenas 0,04 unidades menor que a correlação entre os valores obtidos entre $\mathrm{MA} \mathrm{e}_{\mathrm{AMT}}$, que utilizaram até cinco lactações da vaca para predizer o seu valor genético.

Ao se analisarem as correlações entre os valores genéticos obtidos pelos diversos procedimentos que utilizaram o índice de seleção ( $\mathrm{I}_{\mathrm{AMT}}, \mathrm{I}_{\mathrm{ATT}}, \mathrm{I}_{\mathrm{A} 11}$ e $\mathrm{I}_{\mathrm{PMT}}$ ), observou-se que as correlações variaram de 0,88 a 0,99 , dependendo da quantidade de informações utilizadas no índice e da comparação entre procedimentos (Tabela 5). Estas correlações foram idênticas às obtidas por SPIKE e FREEMAN (1978), que utilizaram procedimentos semelhantes aos do índice de seleção.

$\mathrm{Na}$ Tabela 6 os coeficientes de correlação são apresentados com base em três classes de confiabilidade das predições obtidas pelo I. Embora as correlações para a classe intermediária (confiabilidade entre 0,50 e 0,60 ) não tenham, em geral, valores intermediários às outras duas classes (entre 0,40 e 0,50 e entre 0,60 e 0,70 ), como se esperava, observou-se que para confiabilidades mais elevadas $(0,60$ e 0,70$)$ as correlações obtidas entre os valores genéticos preditos pelo MA e MA1 com os valores preditos pelos índices de seleção foram superiores a 0,83 , à exceção do I. Estes resultados, embora ligeiramente inferiores aos obtidos por NORMAN et al. (1991), para correlações de 0,90 a 0,96 entre os valores genéticos preditos pelo modelo animal e um índice de seleção, sugerem que os índices de seleção utilizando-se as avaliações genéticas dos touros por outro procedimento que não o modelo das companheiras contemporâneas podem ser alternativa viável, quando não se dispõe de estrutura computacional adequada.

Outro fator importante na escolha do procedimento a ser adotado é a avaliação do grau de erro associado às estimativas pelos diferentes procedimentos e como este erro pode influir na seleção das melhores vacas. Com o objetivo de avaliar esse erro, assumiuse que as predições dos valores genéticos obtidos pelo MA seriam as mais adequadas (HENDERSON, 1973, 1988). Assim, calculou-se o desvio médio absoluto dos valores obtidos em cada um dos procedimentos com aqueles obtidos pelo MA. Na Tabela 7 é apresentada a porcentagem de indivíduos cujo desvio absoluto se encontra dentro de determinada classe de desvio médio, para cada procedimento. Admitindo-se que um desvio médio absoluto de até $100 \mathrm{~kg}$ em relação às estimativas pelo MA, cuja média foi de $109 \mathrm{~kg}$ (Tabela 3), seja 
Tabela 6 - Coeficientes de correlação de valores (acima da diagonal) e correlação de ordem (abaixo da diagonal) entre os valores genéticos preditos por diferentes procedimentos ${ }^{1}$ para diferentes valores de confiabilidade do índice de seleção

Table 6 - Correlation coefficient of values (upperdiagonal) and rank correlation (lower diagonal) among genetic values predicted by different procedures ${ }^{1}$ for different values of accuracy by selection index

\begin{tabular}{|c|c|c|c|c|c|c|c|}
\hline \multirow{2}{*}{$\begin{array}{l}\text { Procediment } \\
\text { Procedure }\end{array}$} & \multicolumn{7}{|c|}{$\begin{array}{l}\text { Procedimento } \\
\text { Procedure }\end{array}$} \\
\hline & MA & MA1 & I & $\mathrm{I}_{\mathrm{AMT}}$ & $\mathrm{I}_{\mathrm{ATT}}$ & $\mathrm{I}_{\mathrm{A} 11}$ & $\mathrm{I}_{\mathrm{PMT}}$ \\
\hline & \multicolumn{7}{|c|}{$\begin{array}{l}\text { a) Confiabilidade pelo I entre } 0,40 \text { e } 0,50 \\
\text { Accuracy by I between .40 and .50 }\end{array}$} \\
\hline MA & 1,0 & 0,87 & 0,67 & 0,80 & 0,79 & 0,76 & 0,80 \\
\hline MA1 & 0,85 & 1,0 & 0,66 & 0,74 & 0,73 & 0,80 & 0,74 \\
\hline I & 0,65 & 0,64 & 1,0 & 0,92 & 0,93 & 0,95 & 0,93 \\
\hline & 0,79 & 0,71 & 0,93 & 1,0 & 0,99 & 0,97 & 0,98 \\
\hline & 0,77 & 0,70 & 0,94 & 0,99 & 1,0 & 0,98 & 0,99 \\
\hline & 0,73 & 0,76 & 0,95 & 0,97 & 0,97 & 1,0 & 0,97 \\
\hline \multirow{3}{*}{$\mathrm{I}_{\mathrm{PMT}}$} & 0,79 & 0,71 & 0,91 & 0,99 & 0,99 & 0,97 & 1,0 \\
\hline & \multirow{2}{*}{\multicolumn{7}{|c|}{$\begin{array}{l}\text { b) Confiabilidade pelo I entre } 0,50 \text { e } 0,60 \\
\text { Accuracy by I between } .50 \text { and } .60\end{array}$}} \\
\hline & & & & & & & \\
\hline MA & 1,0 & 0,82 & 0,75 & 0,78 & 0,78 & 0,75 & 0,80 \\
\hline MA1 & 0,81 & 1,0 & 0,69 & 0,74 & 0,72 & 0,80 & 0,74 \\
\hline & 0,71 & 0,66 & 1,0 & 0,87 & 0,88 & 0,89 & 0,88 \\
\hline & 0,78 & 0,71 & 0,89 & 1,0 & 0,98 & 0,94 & 0,99 \\
\hline $\mathrm{I}_{\mathrm{ATT}}$ & 0,76 & 0,69 & 0,89 & 0,98 & 1,0 & 0,93 & 0,98 \\
\hline $\mathrm{I}_{\mathrm{A} 11}$ & 0,73 & 0,78 & 0,87 & 0,94 & 0,93 & 1,0 & 0,94 \\
\hline \multirow{3}{*}{$\mathrm{I}_{\mathrm{PMT}}$} & 0,78 & 0,71 & 0,87 & 0,99 & 0,98 & 0,93 & 1,0 \\
\hline & \multirow{2}{*}{\multicolumn{7}{|c|}{$\begin{array}{l}\text { c) Confiabilidade pelo I entre } 0,60 \text { e } 0, \\
\text { Accuracy by I between } .60 \text { and } .70\end{array}$}} \\
\hline & & & & & & & \\
\hline MA & 1,0 & 0,89 & 0,75 & 0,87 & 0,86 & 0,83 & 0,87 \\
\hline MA1 & 0,89 & 1,0 & 0,77 & 0,84 & 0,84 & 0,88 & 0,84 \\
\hline & 0,74 & 0,76 & 1,0 & 0,80 & 0,79 & 0,81 & 0,81 \\
\hline $\mathrm{I}_{\mathrm{AMT}}$ & 0,86 & 0,83 & 0,81 & 1,0 & 0,98 & 0,96 & 0,99 \\
\hline $\mathrm{I}_{\mathrm{ATT}}$ & 0,85 & 0,82 & 0,80 & 0,99 & 1,0 & 0,96 & 0,99 \\
\hline $\mathrm{I}_{\mathrm{A} 11}$ & 0,82 & 0,86 & 0,80 & 0,96 & 0,95 & 1,0 & 0,96 \\
\hline $\mathrm{I}_{\mathrm{PMT}}$ & 0,86 & 0,83 & 0,80 & 0,99 & 0,99 & 0,96 & 1,0 \\
\hline
\end{tabular}

${ }^{1}$ Vide Tabela 3.

${ }^{1}$ As Table 3.

admissível, verificou-se que apenas 74,9\% dos indivíduos estariam nesta situação, se as avaliações genéticas fossem pelo MA1 (Tabela 7). Caso as avaliações genéticas fossem obtidas por outros procedimentos (I, $\mathrm{I}_{\mathrm{AMT}}$, $\mathrm{I}_{\mathrm{ATT}}, \mathrm{I}_{\mathrm{A} 11}$ ou $\mathrm{I}_{\mathrm{PMT}}$ ), os percentuais variariam de 61,9(I) a, no máximo, $67,4 \%\left(\mathrm{I}_{\mathrm{AMT}}\right.$ e $\left.\mathrm{I}_{\mathrm{A} 11}\right)$.

Outro aspecto importante a ser considerado está relacionado com a seleção dos animais. Na Tabela 8 são apresentadas as porcentagens de coincidência na seleção de vacas pelo modelo animal (MA) e pelos demais procedimentos. Assim, considerando-se o modelo animal (MA) como o ideal para predizer os valores genéticos para se selecionarem vacas para serem mães das futuras gerações, verificou-se que a seleção das $20 \%$ melhores fêmeas avaliadas pelo MA significaria escolher as mesmas vacas em mais de
Tabela 7 - Desvio médio absoluto das estimativas dos valores genéticos pelos diferentes procedimentos $^{1}$ em relação à avaliação pelo modelo animal (MA)

Table 7 - Mean absolute deviation of genetic values estimated by different procedures ${ }^{1}$ in relation to the genetic values estimated by animal model (MA)

Classe de desvio Porcentagem de indivíduos dentro médio absoluto $(\mathrm{kg}) \quad$ das classes de desvio

Class of average absolute Percentage of individual

standard deviation within a deviation class

\begin{tabular}{|c|c|c|c|c|c|c|}
\hline & MA1 & I & & $\overline{\mathrm{I}_{\mathrm{ATT}}}$ & $\mathrm{I}_{\mathrm{A} 11}$ & $\mathrm{I}_{\mathrm{PMT}}$ \\
\hline$<50$ & 61,0 & 52,3 & 55,9 & 54,8 & 53,9 & 56,0 \\
\hline $51-100$ & 13,9 & 9,6 & 11,5 & 11,6 & 11,5 & 11,4 \\
\hline $101-150$ & 10,8 & 8,9 & 10,1 & 9,7 & 9,6 & 10,1 \\
\hline $151-200$ & 6,3 & 7,7 & 7,6 & 7,6 & 7,2 & 7,7 \\
\hline $201-300$ & 5,6 & 10,5 & 8,6 & 9,4 & 10,5 & 8,7 \\
\hline $301-400$ & 1,6 & 6,7 & 3,5 & 3,9 & 4,1 & 3,5 \\
\hline $401-500$ & 0,5 & 2,8 & 1,5 & 1,7 & 1,8 & 1,5 \\
\hline$>500$ & 0,3 & 1,6 & 1,2 & 1,3 & 1,3 & 1,2 \\
\hline
\end{tabular}

1Vide Tabela 3.

${ }^{1}$ As Table 3.

$72 \%$ dos casos, exceto para as escolhidas com base nas avaliações pelo I. Se as avaliações fossem também utilizadas para eliminar todas as vacas com valores genéticos negativos, observou-se que menos de $7 \%$ seriam erroneamente eliminados, em conseqüência da utilização de outro procedimento de avaliação diferente do modelo animal. Todavia, se a eliminação for do terço inferior, verifica-se que, independentemente do procedimento adotado para avaliar as vacas, nenhuma seria eliminada erroneamente.

O principal objetivo de qualquer programa de seleção é obter maior ganho genético $\left(\Delta \mathrm{G}=\mathrm{r}_{\mathrm{m}} \mathrm{D} \sigma_{\mathrm{m}}\right)$, o qual é função da confiabilidade $\left(\mathrm{r}_{\mathrm{m}}\right)$ com que se avaliam os animais, do desvio-padrão das predições genéticas $\left(\sigma_{m}\right)$ e da intensidade de seleção(D). Assim, usando-se os valores de $r_{m}$ e $\sigma_{m}$ apresentados na Tabela 3, foram obtidas as seguintes estimativas de ganho genético, quando $20 \%$ melhores vacas foram selecionadas $(D=1,4)$ para produzirem descendentes para a próxima geração, segundo os diferentes procedimentos: $270 \mathrm{~kg}$ para MA, $219 \mathrm{~kg}$ para MA1, $216 \mathrm{~kg}$ para I, $248 \mathrm{~kg}$ para $\mathrm{I}_{\mathrm{AMT}}, 244 \mathrm{~kg}$ para $\mathrm{I}_{\mathrm{ATT}}$, $229 \mathrm{~kg}$ para IA 11 e $247 \mathrm{~kg}$ para IPMT. Estes valores indicam que na pior das hipóteses, escolhendo-se as vacas pelo I, obtiveram-se $80 \%$ (216/270) do ganho genético, que seria obtido caso a seleção fosse baseada no MA, e cerca de $91 \%$ do ganho pelas estimativas do MA, caso a seleção fosse por um dos procedimentos $\mathrm{I}_{\mathrm{AMT}}, \mathrm{I}_{\mathrm{ATT}}$ ou $\mathrm{I}_{\mathrm{PMT}}$. 
Rev. bras. zootec.

Tabela 8 -Porcentagem de vacas selecionadas pelo modelo animal (MA) e sua correspondência com os demais procedimentos ${ }^{1}$ usados para a avaliação genética

Table 8 - Percentage of cows selected byanimal model(MA) and its relationship with the others procedures ${ }^{1}$ used forgenetic evaluation

Porcentagem selecionada Porcentagem de coincidência pelo MA pelos demais procedimentos

Percentage selected Percentage of coincidence by $M A \quad$ by the others procedures

\begin{tabular}{lrrrrrr} 
& MA1 & \multicolumn{1}{c}{$\mathrm{I}$} & $\mathrm{I}_{\text {AMT }}$ & $\mathrm{I}_{\text {ATT }}$ & $\mathrm{I}_{\text {A11 }}$ & $\mathrm{I}_{\text {PMT }}$ \\
\hline 5 & 63,3 & 52,1 & 58,1 & 57,0 & 54,6 & 58,4 \\
10 & 71,6 & 59,9 & 67,4 & 65,9 & 64,0 & 67,1 \\
15 & 74,6 & 65,0 & 71,7 & 70,0 & 68,5 & 71,8 \\
20 & 78,0 & 68,0 & 74,9 & 73,0 & 72,2 & 75,0 \\
30 & 83,0 & 75,9 & 81,3 & 80,6 & 79,3 & 81,3 \\
40 & 87,7 & 84,6 & 86,9 & 86,7 & 85,1 & 87,0 \\
50 & 93,9 & 93,2 & 94,6 & 94,2 & 93,4 & 94,6 \\
60 & 100,0 & 100,0 & 100,0 & 100,0 & 100,0 & 100,0 \\
\hline 1Vide Tabela 3. & & & & & &
\end{tabular}

${ }^{1}$ As Table 3.

\section{Conclusões}

As avaliações genéticas pelo modelo animal, no qual se incluíram até cinco lactações da vaca, foram mais precisas e proporcionariam maior ganho genético, se fossem utilizadas para avaliar e selecionar vacas, que as avaliações pelos índices de seleção.

As avaliações genéticas obtidas pelos índices de seleção que utilizaram valores genéticos dos touros obtidos pelo modelo animal foram menos precisas, mas proporcionariam $90 \%$ do ganho genético que seria obtido com a utilização do modelo animal.

A eliminação das vacas do terço inferior da população utilizando-se as avaliações genéticas proporcionaria o descarte das mesmas vacas, independente do procedimento adotado para avaliá-las.

\section{Referências Bibliográficas}

BOLDMAN, K.G., KRIESE, L.A., VAN VLECK, L.D. et al. 1995. A manual for use of MTFREML: a set of programs to obtain estimates of variance and covariances. Lincoln: Departament of Agriculture/Agriculture Research Service. $120 \mathrm{p}$.

CONOVER, W.J. 1980. Pratical non parametric statistics. 2 ed. New York: John Wiley. 493p.

DICKINSON, F.N., NORMAN, H.D., POWELL, R.L. et al. 1976. Procedures used to calculate the USDA-DHIA modified comtemporary comparison. In: (USDA Prod. Res. Rep., n. 165, p.18). Washington.

DOMMERHOLT, J. 1980. Developments in methods of estimating breeding value in bulls and cows. Bedrijfsontwikkeling. 11:116-170.

DOMMERHOLT, J. 1982. Sire and cow evaluations program. Coronel Pacheco: Embrapa-CNPGL. 42p (mimeo).

FALCONER, D.S. 1981. Introdução à genética quantitativa.
Viçosa: Imprensa Universitária. 279p.

HENDERSON, C.R. 1973. Sire evaluation and genetic trends. In: ANIMAL BREEDING AND GENETICS SYMPOSIUM, 1, 1973, Champaing. Proceedings... Menasha: American Society of Animal Science, p.41.

HENDERSON, C.R. 1975. Use of all relatives in intraherd prediction of breeding values and producing abilities. $J$. Dairy Sci., 58:1910-1916.

HENDERSON, C.R. Theoretical bases and computational methods for a number of differents animal models. In: ANIMAL MODEL WORKSHOP, 1, 1988, Edmonton. Proceedings... Edmonton, 1988.

HILL, W.G., SWANSON, G.J.T. 1983. A selection index for dairy cows. Anim. Prod., 37:313-319,

MOEN, R.A. 1978. A note on a special case of estimation of breeding value. Acta Agric-Scand., 28:148-150.

NORMAN, H.D., POWELL, R.L, WIGGANS, G.R.1991. Comparison of genetic evaluations from animal model and modified contemporary comparison. J. Dairy Sci., 74:23092316.

POWELL, R.L., NORMAN, H.D., WEINLAND, B.T. 1983. Cow evaluation at different milk yields of herds. J. Dairy Sci., 66:148-154.

SPIKE, P.L., FREEMAN, A.E. 1978. Prediction of genetic differences among herds with estimates of breeding values. J. Dairy Sci., 61:1476-1482.

VAN VLECK, L.D. 1992. Animal model for bull and cow evaluation. In: LARGE DAIRY HERD MANAGEMENT SYMPOSIUM. Gainesville, p.1-31.

VAN VLECK, L.D. 1993. Selection index and introduction to mixed model methods. 1 ed. Florida: CRC Press Inc., 481 p.

VERNEQUE, R.S. Procedimentos numéricos e estimação de componentes de co-variância em análise multivariada pelo método da máxima verossimilhança restrita: modelos mistos aplicados ao melhoramento animal. Piracicaba, SP, ESALQ, 1994. 155p. Tese (Doutorado em Bioestatística) Escola Superior de Agricultura Luiz de Queiroz, 1994.

Recebido em: $13 / 02 / 98$

Aceito em: 17/12/98 